\title{
Narrativa
}

\section{Tradotto dall'Italia. Narrativa italiana contemporanea nelle traduzioni ceche dopo il 2000}

\section{Alice Flemrová}

\section{(2) OpenEdition}

\section{Journals}

\section{Edizione digitale}

URL: https://journals.openedition.org/narrativa/840

DOI: $10.4000 /$ narrativa. 840

ISSN: 2804-1224

\section{Editore}

Presses universitaires de Paris Nanterre

\section{Edizione cartacea}

Data di pubblicazione: 1 décembre 2016

Paginazione: 113-127

ISBN: 978-2-84016-266-7

ISSN: 1166-3243

Notizia bibliografica digitale

Alice Flemrová, «Tradotto dall'Italia. Narrativa italiana contemporanea nelle traduzioni ceche dopo il 2000», Narrativa [Online], 38 | 2016, online dal 01 janvier 2022, consultato il 22 février 2022. URL: http://journals.openedition.org/narrativa/840 ; DOI: https://doi.org/10.4000/narrativa.840

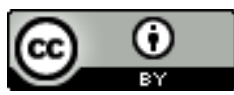

Narrativa est mise à disposition selon les termes de la Licence Creative Commons Attribution 4.0 International. 


\title{
Tradotto dall'Italia. Narrativa italiana contemporanea nelle traduzioni ceche dopo il 2000
}

\author{
PICCOLA PREMESSA STORICA
}

Come ha già segnalato Chiara Mengozzi ${ }^{1}$, dopo il 1989 il mercato dei libri cecoslovacco - che nel 1993, con la scissione del paese, si è diviso in due mercati autonomi, quello ceco e quello slovacco ${ }^{2}$ - subisce una grande e profonda ma nello stesso tempo disordinata e selvaggia trasformazione le cui conseguenze (ma forse dovremmo parlare piuttosto di effetti collaterali) si notano tutt'oggi, segnando il periodo che abbiamo deciso di analizzare, ossia gli Anni Zero e Dieci3

Senza entrare nei dettagli della situazione nel campo dell'editoria cecoslovacca prima del 1989, si devono sottolineare almeno due aspetti:

1. Cfr. Mengozzi, Chiara, "Le leggi del mercato e le preferenze dei lettori. Ipotesi sulla circolazione e il successo della narrativa italiana ultra-contemporanea in Repubblica Ceca", in questo volume.

2. All'epoca, gli abitanti della Cecoslovacchia erano in gran parte bilingui. Col passare del tempo si è manifestata anche una scissione linguistica tra i due popoli. Se ancora nel corso degli anni Novanta gli slovacchi leggevano libri cechi (ciò vale anche per i libri tradotti in ceco) e i cechi, anche se in minor numero, leggevano quelli slovacchi, oggi i lettori cechi e slovacchi si limitano piuttosto al mercato dei libri "nazionale" e sono sempre più frequenti i casi di traduzioni da una lingua all'altra.

3. Per informazioni sulla situazione attuale riguardo il mercato ceco dei libri si rimanda a TrÁvNíčEK, Jiří, Reading Bohemia Readership in the Czech Republic at the beginning of the 21th century, Brno, Akropolis, 2015; vengono qui presentati i risultati di tre grandi inchieste sul mondo della lettura nella Repubblica Ceca, realizzate dall'Istituto di letteratura ceca dell'Accademia delle Scienze in collaborazione con la Biblioteca nazionale negli anni 2007, 2010 e 2013. La cultura dei lettori nella Repubblica Ceca risulta "a reading culture that generates few non-readers, but not too many above average readers either (whose number is falling in any case), and yet with a fairly large number of readers who are high above the average (i.e. "passionate" readers)" (p. 87). 
- La censura ideologica e politica ostacolava la pubblicazione di certi autori e di certi libri (possiamo riportare l'esempio di Primo Levi: I sommersi e i salvati vengono pubblicati in ceco per la prima volta dalla casa editrice Index a Colonia nel 1989; Se questo è un nomo esce in ceco solo nel 19914. Considerando il ritardo con cui Levi è giunto al pubblico ceco, risulta più facile capire perché sia ritenuto ancora oggi un autore "contemporaneo".

- Gli autori non proibiti venivano comunque interpretati, o piuttosto misinterpretati, in base al prisma ideologico-politico oppure si taceva quella parte della loro opera che poteva risultare scomoda o addirittura inaccettabile per i criteri della morale e dell'estetica socialista. L'autore italiano più tradotto e letto in Cecoslovacchia durante i quarant'anni del regime è stato Alberto Moravia ${ }^{6}$, autore di cui però il pubblico ceco e slovacco ignora a tutt'oggi la produzione saggistica e quella teatrale. Andrebbe approfondita la ricerca sulle motivazioni della sua indiscussa popolarità nella Cecoslovacchia di allora, riconsiderando il ruolo che in quegli anni aveva la grande richiesta di scritti su tematiche a sfondo erotico, richiesta rimasta insoddisfatta dal mercato editoriale cecoslovacco prima del 1989.

Nel momento in cui sono venuti a sparire i divieti ideologici, hanno cominciato a imporsi, dopo il breve periodo dell'entusiasmo idealista, i dettami del mercato. Gli editori si sentivano sia in debito verso gli autori ostacolati e censurati dopo il 1948 - tante erano le lacune da colmare - sia bramosi di aprirsi all'attualità, di mettersi al passo con i tempi, di far leggere al pubblico ceco le novità, di entrare nel mercato globale dei libri.

Il discorso fatto per la politica editoriale riguarda anche i programmi universitari (o scolastici in genere) nel periodo che va dal 1948 al 1989. Dati i limiti di lunghezza del presente articolo non ci spingiamo oltre. Segnaliamo soltanto che i corsi universitari dedicati alla letteratura contemporanea e a quella ultra-contemporanea fanno la loro comparsa solo all'inizio degli Anni Zero e solo come facoltativi. Tuttavia abbastanza presto essi trovano spazio nei programmi curricolari. Il crescente interesse degli studenti per la narrativa e per la saggistica

4. L’unico libro uscito in ceco prima del 1989 è Il sistema periodico: Levi, Primo, Prvky života, Praha, Odeon, 1975. In molti cataloghi di biblioteca risulta sorprendentemente inserito nella categoria "fantascienza e fantasy".

5. Alcuni degli scrittori cechi interpellati da me e da Chiara Mengozzi (cfr. l'allegato 2) hanno citato Primo Levi tra gli autori italiani ultracontemporanei.

6. Vedi Flemrová, Alice, "La fortuna di Alberto Moravia in Cecoslovacchia. L'autore conosciuto di più non sempre è conosciuto bene", in FAVARO, Angelo (a cura di), Alberto Moravia e la Ciociara. Letteratura. Storia. Cinema III, Avellino, Sinestesie, 2015, pp. 93-102. 
italiana contemporanea e ultra-contemporanea è confermato dai temi che si possono ricavare dai depositi elettronici delle tesi di laurea discusse negli ultimi dieci anni nelle università ceche e morave ${ }^{7}$.

\section{UNA FIGURA CHIAVE}

Si possono evidenziare due livelli fondamentali per la capacità di scoprire e avviare un autore a una durata (catalogo), a un futuro squisitamente editoriale, che presuppone naturalmente una qualità per dirla qui in breve. [...] I due livelli in sostanza sono: il livello della sperimentazione, scoperta, maieutica, tipico del consulente o direttore di collana o editor [...] e il livello delle pratiche, della macchina, dei processi produttivo-promozionali-distributivi, tipico dell'editore imprenditore o insomma dell'editore tout court (e/o dei dirigenti e funzionari editoriali). Va aggiunto che la prefigurazione di una durata e di un pubblico nei casi migliori è ben presente a entrambi i livelli ${ }^{8}$.

Nel libro da cui abbiamo tratto suddetta citazione, Ferretti traccia la storia dei rifiuti editoriali e rivolge l'attenzione ai casi di manoscritti rifiutati e successivamente rivalutati, per diventare poi dei libri a tutti gli effetti. Dalla ricca casistica presentata nel volume emerge in modo chiaro un'informazione che può sembrare a prima vista banale e scontata, ma di cui in realtà spesso ci si dimentica: nella fortuna dei libri interviene sempre, anche nel contesto del postumanesimo, il fattore umano. Se tentiamo di applicare i due livelli di cui scrive Ferretti all'attuale realtà dell'editoria ceca, ci rendiamo immediatamente conto che il livello della sperimentazione (perlomeno nel campo dell'importazione editoriale) risente della mancanza di protagonisti. Se prima del 1989 le case editrici impiegavano nelle proprie redazioni folte schiere di editor laureati in filologia o in lingue moderne che si occupavano delle letterature nazionali di propria competenza ${ }^{9}$, oggi gli editor e i curatori di un testo sono diventati quasi una specie a rischio di estinzione. Senza voler accentuare il ruolo delle letterature nazionali,

7. Tra i nomi che appaiono più spesso possiamo citare Ammaniti, Baricco, Benni, Camilleri, Eco, Tabucchi, ma si è scritto anche su Atzeni, Saviano, Piperno, Ballestra, Lucarelli, Nove, Mastrocola. Cfr. https://is.cuni.cz/webapps/zzp/search/?tab_ searchas =basic\&lang=en (Università di Carlo IV di Praga) o http://is.muni.cz/thesis / (Università di Masaryk di Brno).

8. Ferretti, Gian Carlo, Siamo spiacenti. Controstoria dell'editoria italiana attraverso $i$ rifiuti, Milano, Mondadori, 2012, p. 5.

9. Come abbiamo già fatto notare non avevano carta bianca. Spesso però cercavano di aggirare i censori per riuscire a far pubblicare libri importanti; e nonostante gli ostacoli, si sforzavano di non perdere di vista la letteratura dell'area di cui si occupavano. 
nella scomparsa degli editori e dei redattori specializzati vediamo un grave problema pratico: la mancanza di figure che parlino le lingue a diffusione limitata riduce il concetto multilinguistico e multiculturale della letteratura mondiale a uno spazio delimitato dalle "conquiste e scoperte" dell'inglese come lingua veicolare $^{10}$. Le case editrici, almeno quelle più serie, sostituiscono perciò le figure fisse, interne, con vari collaboratori esterni. Essendo l'accessibilità ai testi stranieri legata alle competenze linguistiche, è logico che tra questi collaboratori esterni si trovi un alto numero di traduttori. Il traduttore diventa così una figura chiave che opera al livello "della sperimentazione editoriale". La traduzione stessa è solo una delle tante attività che il traduttore deve svolgere per poter vedere un'idea diventare libro. Le figure eminenti di intellettuali e di letterati operanti a questo primo livello avevano sempre una "visione", spesso molto soggettiva e individuale, basata sul proprio gusto di lettore, cui subordinavano le proprie strategie editoriali ${ }^{11}$. Una visione personale non manca nemmeno oggi; essa deve però di continuo confrontarsi, o scontrarsi, con le figure che rappresentano il secondo livello, quello pratico, e che sono oramai tutte coinvolte nella caccia al best seller.

\section{TRADUTTORE-TUTTOFARE ${ }^{12}$}

L'associare più ruoli, lavori e mestieri in una figura empirica ha ovviamente sia i lati positivi, sia quelli negativi. Un docente universitario-traduttore-scoutconsulente editoriale-editor-recensore non è nella Repubblica Ceca una rarità, soprattutto se la persona in questione insegna una delle letterature considerate "minori" e/o traduce da una lingua minoritaria.

Tra i benefici possiamo sottolineare la possibilità di mettere in contatto l'ambiente accademico con il mondo dell'editoria commerciale: il risultato di tale

10. Spazio davvero limitato, visto che "negli Usa e in Inghilterra solo il 3\% dei libri in commercio è tradotto, percentuale che per la fiction si abbassa a un drammatico 0,7\%"; Speciale, Andreina, "Tre percento, la traduzione di chi non traduce", in Spinazzola, Vittorio (a cura di), Tirature'16, Il mondo da tradurre, Milano, il Saggiatore, 2016, p. 31.

11. Cfr. Cadioli, Alberto, Letterati editori. L’industria culturale come progetto, Milano, il Saggiatore, 2003 e ID., Le diverse pagine. Il testo letterario tra scrittore, editore, lettore, Milano, il Saggiatore, 2012.

12. Mi permetterò di parlare dell'importanza, del ruolo e delle (im)possibili strategie del traduttore nella Repubblica Ceca basandomi soprattutto sulla mia esperienza personale di docente universitario il cui secondo mestiere è la traduzione letteraria. Le informazioni più generali sulle molteplici attività di un traduttore nella Repubblica Ceca dopo il 2000 sono tratte anche dalle esperienze dei miei colleghi docenti-traduttori. 
incontro non deve essere per forza un ossimoro, esso anzi può aiutare tutti e due i mondi a uscire dai propri orizzonti restrittivi. Una lunga e felice collaborazione può permettere al traduttore di convincere l'editore a pubblicare ogni tanto un autore sconosciuto anche a costo di subire perdite finanziarie. Di solito usando il metodo del baratto: io traduco un altro romanzo del bestsellerista che ho scoperto per te e tu pubblichi il romanzo di un esordiente che probabilmente non diventerà mai un best seller ma che io ritengo importante, anche se il messaggio dovesse arrivare solo a happy few.

Tra gli svantaggi va in primo luogo osservata la quasi totale assenza di un dialogo e di un feedback. Da questo punto di vista la situazione è molto peggiorata negli Anni Dieci - il genere della recensione sta diventando di nicchia, coltivato da pochi, e soprattutto sta degenerando in pubblicità occulta se non addirittura in autopromozione.

Ultimamente capita di leggere migliori o quanto meno più interessanti recensioni (spesso hanno anche un livello stilistico più alto) su alcuni blog o su pagine web per appassionati di letteratura dove di solito intervengono persone "non addette ai lavori" che non nelle pagine culturali delle grandi testate. La critica letteraria viene ancora coltivata da alcune riviste letterarie e culturali cartacee o elettroniche, ma si tratta di pubblicazioni decisamente di nicchia, benché quelle elettroniche siano in grado di raggiungere un numero di lettori già significativo. Se torniamo alle pagine curate da non addetti ai lavori, bisognerebbe vederle in fin dei conti come un segnale positivo. La stessa esistenza dei forum e delle comunità di lettori dimostra che ci sono ancora persone che non solo leggono libri, ma che hanno voglia di esprimere i propri giudizi e di discuterne. La scoperta di avere un pubblico, composto pure da "venticinque lettori”" è nei tempi che corrono per il traduttore una vera soddisfazione.

Cerchiamo ora di riassumere le attività che spesso deve svolgere un traduttore: mantiene contatti con le case editrici all'estero per essere sempre informato sulle novità editoriali ed avere testi a disposizione; partecipa a fiere internazionali del libro dove fa le veci dello scout, legge un'enorme quantità di testi, ne fa una preselezione, anch'egli alla ricerca di un possibile best seller o long seller, ma nello stesso tempo propone anche testi e autori che ritiene significativi per qualità estetiche e/o etiche; scrive giudizi sui libri prescelti, a volte traduce brevi brani del testo a mo" di "assaggio"; è sempre in cerca di sovvenzioni, sponsor, borse, collabora attivamente con case editrici nella fase di richie- 
sta di un supporto finanziario ${ }^{13}$; è in cerca dell'editor del libro che ha tradotto, perché, come si è detto poco sopra, le case editrici ceche hanno diminuito al massimo il personale fisso; in questo momento nell'editoria ceca lavora stabilmente solo un redattore che conosce l'italiano (peraltro solo come quarta lingua); traduce; spesso scrive la postfazione o la prefazione del libro tradotto, soprattutto se si tratta di un autore sconosciuto al pubblico, cura la quarta di copertina, scrive il riassunto da inviare ai librai e ai distributori ${ }^{14}$; collabora con istituti di cultura e/o altre istituzioni in occasione dell'invito dello scrittore e nell'organizzazione della sua visita, aiuta a promuovere il libro sui media; in alcuni casi si prende cura anche delle recensioni: le fa scrivere ai propri studenti, agli amici.

La loro unica attività remunerata è tuttavia la traduzione. Alcune case editrici retribuiscono anche i giudizi del lettore e le postfazioni, ma sono sempre meno. Da questo si può dedurre che se esistono ancora libri tradotti da lingue e letterature medio-piccole sul mercato ceco, è perché i traduttori fanno la parte del leone in questa impresa.

\section{WROTE IN ITALY: CHE COSA OFFRE IL MERCATO CECO?}

Prima di vedere che cosa della letteratura italiana post-2000 sia stato concretamente tradotto in ceco, ci soffermeremo brevemente sulla questione dell'esistenza o meno di strategie per "creare" un best seller e sulla possibilità di importare un successo realizzato all'estero (non solo nel paese d'origine del libro) anche nel contesto interno. Nella fase di selezione di un libro possiamo individuare alcuni fattori influenti che le case editrici prendono in considerazione: successo internazionale, premi e riconoscimenti importanti, vendite all'estero, adattamento cinematografico esistente o almeno in corso, tema o genere

13. Cfr. allegato 1; di solito, gli editori cechi presentano la richiesta di sovvenzione in italiano, e chi redige i documenti è in tal caso il traduttore. Inoltre, il bando per la richiesta di contributi per la traduzione di opere letterarie pubblicato dal Ministero di Affari Esteri, diffuso degli Istituti Italiani di Cultura, è in italiano.

14. Per non sbilanciare troppo il fragile equilibrio tra le due qualità che deve avere un libro di belle lettere, ossia quella di essere un'opera d'arte e al tempo stesso una merce, è sempre meglio che lo faccia il traduttore, che ha letto il libro, e non un esperto delle strategie di marketing della promozione del libro che non conosce il prodotto da vendere. Tradizionalmente questo compito spettava ai rappresentanti del primo livello, i letterati-editori spesso mantenevano il dialogo con i lettori tramite i testi sul risvolto del libro che avevano curato, scrivendoli in modo soggettivo ed esprimendo il proprio gusto e le proprie opinioni. Cfr. CADIOLI, Le diverse pagine, cit., capitolo "Gli scritti editoriali che accompagnano il testo", pp. 214-23. 
"che vende". Nella fase promozionale: visita dell'autore/autrice ${ }^{15}$, interviste e recensioni sui quotidiani più diffusi, raccomandazione da parte di un famoso personaggio "mediatico". Il libro acquisisce così l'importanza di un testo che bisogna assolutamente leggere per non sfigurare. Nella valutazione bisogna tuttavia tener conto di almeno un'imprevedibile variabile ignota. In più, il mercato è saturo ${ }^{16}$, e non ci riferiamo tanto alla concorrenza in termini di qualità, quanto alla mole di libri di fronte ai quali il lettore non sa come orientarsi.

Nella tabella che segue (allegato 1) si trovano i libri degli scrittori italiani (o di quelli che scrivono in italiano) attivi anche dopo il 2000, che sono stati tradotti in ceco negli Anni Zero o Dieci ${ }^{17}$. L'elenco presentato vuole essere illustrativo e non esaustivo, si tratta di una selezione, sono stati tralasciati libri per bambini, pubblicazioni religiose, saggi e libri divulgativi sulla storia e sulla storia dell'arte ${ }^{18}$. La poesia non è stata né tralasciata né omessa: manca semplicemente. Per arrivare a risultati più convincenti bisognerebbe considerare ulteriori dati importanti: tirature, vendite, quantità e qualità delle recensioni e soprattutto andrebbe valutata la qualità delle singole traduzioni ${ }^{19}$, perciò ci limiteremo solo a qualche

15. Piacciono autori con una storia particolare alle spalle, per esempio Giordano, presentato come un nerd, Lilin presentato come un cecchino con un passato criminale.

16. Questo risulta anche dall'inchiesta presentata da Trávníček nel libro sopra citato. L'autore lo dice esplicitamente anche in un recente articolo: TrÁvNíčEK, Jiř́i, "Krize? Ale jděte", Host, n. 2, 2016, pp. 20-23: "Dall'ultima inchiesta condotta nel 2013 e orientata appunto sul mercato dei libri risulta infatti che la barriera culturale è più grande di quella economica. Sono troppi i libri, una marea, nella quale ci perdiamo, spesso non sappiamo scegliere", nostra la traduzione.

17. Purtroppo la banca dati della Comunità dei traduttori cechi, http://www. obecprekladatelu.cz, non è stata aggiornata dopo il 2000, almeno per quello che riguarda le traduzioni e i traduttori dall'italiano, così mi sono basata sui dati reperibili sui cataloghi delle case editrici, delle librerie on line e delle biblioteche.

18. Al centro della nostra attenzione si trova la narrativa anche se sappiamo che statisticamente "[essa] copre solo il 16,6\% dell'export, mentre più dell' $80 \%$ dell'attività di cessione diritti esteri riguarda il settore bambini e ragazzi (45\%), la saggistica $(21 \%)$ e il libro illustrato, d'arte, lifestyle, design o fotografia (15\%). Le proporzioni appaiono quindi rovesciate rispetto al peso che la nostra tradizione normalmente accorda alla produzione umanistico-letteraria, e distorte rispetto all'abituale marginalità di alcuni settori, come il libro d'arte e il libro per ragazzi, che insieme pesano non più del $14 \%$ della produzione editoriale italiana"; SPECIALE, Andreina, "L'editoria italiana oltre confine", in Spinazzola, Vittorio (a cura di), Tirature'15, Gli intellettuali che fanno opinione, Milano, il Saggiatore, 2015, pp. 188-89.

19. Va subito precisato che non è sempre l'alta qualità della traduzione (per esempio quella che ottiene riconoscimenti e premi in ambito professionale) a essere apprezzata dal pubblico: il lettore medio non ama molto le traduzioni coraggiose che devono a volte violentare un po' la lingua a favore di una traduzione straniante. Cfr. VENUTI, Lawrence, L'invisibilità del traduttore. Una storia della traduæione [1995], Roma, Armando, 
commento. Alla nostra attenzione non dovrebbe sfuggire l'ultima colonna della tabella. I dati provengono da una banca dati creata da amanti della lettura e della letteratura, una comunità anonima (i membri si registrano in maggior parte con uno pseudonimo) che valuta e commenta i libri che ha letto. Sulla banca dati risultano più dati statistici, nella tabella ci siamo limitati a inserire solo il numero dei lettori che hanno dato una valutazione percentuale al libro. Il numero di membri attualmente registrati è pari a $147.836^{20}$, l'unico scrittore italiano che si è posizionato nella classifica dei cento autori più popolari (su un totale di 90.956) è Umberto Eco (attualmente occupa il cinquantatreesimo posto). Il successo del fenomeno Eco non sorprende, del resto lo scrittore è stato anche più volte nominato nel questionario che abbiamo distribuito ${ }^{21}$. La vox populi difatti sorprendentemente coincide con i risultati del nostro questionario, con talune differenze da parte degli studenti che frequentano i corsi della laurea magistrale e dei dottorandi.

Se ci concentriamo un attimo sul numero delle persone che hanno valutato $i$ libri nella tabella, vediamo che la soglia dei 100 voti è stata superata da Eco con tutti i romanzi presenti, da Giordano con La solitudine di numeri primi, da Brizzi con 100 giorni di felicità, da D'Avenia con Bianca come il latte, rossa come il sangue e da Luca Di Fulvio con tutti e due i romanzi, già mega-best seller in Germania. Il romanzo di Giordano ha raggiunto il numero di voti più alto in assoluto e ciò confermerebbe l'opinione della casa editrice Odeon (che pubblica tra l'altro Murakami, Houellebecq, Palahniuk, Barnes e altri bestselleristi) per la quale l'opera dell'autore italiano rappresenta un chiaro successo commerciale. Lo stesso non si può dire per gli altri due libri dell'autore. L'insuperabilità dell'esordio "di grido" è diventata quasi una regola. D'Avenia e Brizzi, completamente trascurati dai recensori cechi, possono essere considerati come degli esempi della forza del passaparola tra i lettori. Il "buono vincente" sarà nel loro caso il tema: il male incurabile attira sempre. Invece alcuni dei libri di cui si è parlato molto sui media cechi e che sono considerati best seller dai loro editori non arrivano a cento voti e devono accontentarsi di aver raggiunto la soglia dei cin-

1999, p. 201: "La traduzione straniante è la pratica culturale trasgressiva, dal momento che afferma il rifiuto del predominio dei valori linguistici e letterari interni per sviluppare un'affiliazione a quelli marginali e includere culture straniere che sono state escluse a causa della loro resistenza a quegli stessi valori dominanti".

20. Cfr. http://www.databazeknih.cz/statistiky. Nella pagina web sono registrati anche lettori slovacchi.

21. Vedi Mengozzi, Chiara, "Le leggi del mercato...", in questo volume, nota 3 e allegato 2 . 
quanta: tra essi Gomorra di Saviano e Educazione siberiana di Lilin. Ciò può essere spiegato anche dal fatto che i lettori di questo tipo di letteratura frequentano altri siti web o altri gruppi su Internet ${ }^{22}$. Vediamo, inoltre, che tutti i libri con il maggior numero di voti hanno anche valutazioni alte, più dell' $80 \%$, o quantomeno medie: più del $70 \%$. Sul lato opposto della scala valutativa troviamo libri come Per grazia ricevuta o Proleterka. Possiamo chiederci perché i libri della Agus e della Murgia funzionino meglio presso il pubblico ceco del libro della Parrella. Se prescindiamo dall'ipotesi che i lettori preferiscano il romanzo alle novelle, bisogna cercare le risposte nell'ambientazione delle storie o nella lingua dell'autore. La Sardegna delle autrici sopra citate corrisponde all'immagine di una terra mitica ed esotica a differenza della Napoli quotidiana presentata da Valeria Parrella tramite incontri ravvicinati del terzo tipo. La questione della lingua è molto complessa e non può essere liquidata in poche righe. Ripeteremo quanto già osservato: il pubblico non professionale preferisce i libri scritti in un linguaggio che non pone ostacoli alla lettura, che permette al testo di scorrere velocemente. E questo non è il caso della Parrella, né tantomeno di Vasta o di Piperno... Sorprendente è invece lo scarso successo dei libri gialli e dei noir.

Andreina Speciale nell'articolo citato sostiene che

il genere italiano di maggior successo oltreconfine si dimostra senza troppe sorprese il crime novel: soprattutto in quei Paesi beneficiati da un ampio pubblico di lettori forti e curiosi di aprirsi ad altri orizzonti culturali, come quelli di lingua tedesca, spopolano autori come Lucarelli, Carofiglio, Camilleri e ovviamente Saviano, concordemente a un'immagine della nazione italiana che fatica ad andare oltre gli stereotipi ben noti ${ }^{23}$.

Il pubblico ceco non corrisponderebbe in tal caso alla caratteristica dei lettori forti e "curiosi di aprirsi ad altri orizzonti culturali". Dal nostro punto di vista Saviano non andrebbe mescolato con gli autori del crime novel, perché il suo successo, almeno nella Repubblica Ceca, come risulta anche dalle recensioni, è dovuto molto alla sua immagine di testimone oculare, di martire, di giornalista investigativo. Possiamo forse sostenere che il pubblico ceco conserva alcune sue peculiarità e così non tutti i libri di successo italiani (ben accolti anche in Germania, in Polonia, in Francia) diventano best seller anche sul mercato ceco.

22. Va anche ricordato che la cultura ceca dei lettori è "a reading culture with a very high gender barrier - and a large gap between women and men (in favour of women)", TrÁvníčEK, Jiří, Reading Bohemia Readership..., cit., p. 87.

23. Speciale, Andreina, "L'editoria italiana oltre confine", cit., p. 190. 
La Mazzantini, la Ferrante ${ }^{24}$, Volo, la Littizzetto hanno lasciato i cechi piuttosto indifferenti per ora, ma talvolta, sappiamo, basta una nuova edizione - senza che si debba trattare di una nuova traduzione - a cambiare il destino di un libro.

L'esperienza ci insegna che il canone letterario si crea sempre a posteriori, allo stato attuale vediamo quindi che il compito degli editori, dei traduttori e degli editor consiste nello sforzo di pubblicare opere letterarie in traduzione di sufficiente qualità, quantità e variabilità, idealmente in base a una scelta ponderata e sistematica, e non con il diffuso metodo "gratta e vinci", in modo tale da creare un corpus-campione rappresentativo di testi.

Alice Flemrová Univerzita Karlova v Praze

24. Un altro editore ceco pubblicherà la tetralogia L'amica geniale: la ricezione mostrerà se il pubblico ceco è veramente così diverso. 


\section{ALLEGATO 1}

TITOLI ITALIANI TRADOTTI DOPO IL 2001 (solo autori attivi negli Anni Zero e/o Dieci)

\begin{tabular}{|c|c|c|c|c|c|}
\hline AUTORE & TITOLO & $\begin{array}{l}\text { Anno } \\
\text { IT }\end{array}$ & $\begin{array}{c}\text { Anno } \\
\text { CZ }\end{array}$ & $\begin{array}{l}\text { Valutazione } \\
0-100 \% / \\
\text { numero voti }\end{array}$ & $\begin{array}{c}\text { Sovvenzione }^{25} / \\
\text { Editore } \text { ceco }^{26}\end{array}$ \\
\hline Milena Agus & Mal di pietre & 2006 & 2010 & $86 \% / 17$ & Sì / Paseka \\
\hline $\begin{array}{l}\text { Niccolò } \\
\text { Ammaniti }\end{array}$ & $\begin{array}{l}\text { Ti prendo e ti porto } \\
\text { via } \\
\text { Io non ho paura } \\
\text { Io e te }\end{array}$ & $\begin{array}{l}1999 \\
2001 \\
2010\end{array}$ & $\begin{array}{l}2008 \\
2005 \\
2014\end{array}$ & $\begin{array}{l}94 \% / 17 \\
83 \% / 34 \\
72 \% / 13\end{array}$ & $\begin{array}{l}\text { Sì / Havran } \\
\text { Sì / Havran } \\
\text { No / NLN }\end{array}$ \\
\hline $\begin{array}{l}\text { Silvia } \\
\text { Avallone }\end{array}$ & Acciaio & 2010 & 2014 & $54 \% / 14$ & No / Motto \\
\hline $\begin{array}{l}\text { Alessandro } \\
\text { Baricco }\end{array}$ & $\begin{array}{l}\text { Castelli di rabbia } \\
\text { Oceano mare } \\
\text { Seta } \\
\text { City } \\
\text { Mr. Guyn }\end{array}$ & $\begin{array}{l}1991 \\
1993 \\
1996 \\
1999 \\
2011\end{array}$ & $\begin{array}{l}2003 \\
2001 \\
2004 \\
2000 \\
2015\end{array}$ & $\begin{array}{l}83 \% / 14 \\
92 \% / 28 \\
86 \% / 53 \\
69 \% / 13 \\
89 \% / 24\end{array}$ & $\begin{array}{l}\text { No / Eminent } \\
\text { No / Eminent } \\
\text { No / Eminent } \\
\text { No / Volvox } \\
\text { Globator } \\
\text { No / Slovart }\end{array}$ \\
\hline $\begin{array}{l}\text { Stefano } \\
\text { Benni }\end{array}$ & $\begin{array}{l}\text { Bar Sport } \\
\text { Saltatempo } \\
\text { Stranalandia }\end{array}$ & $\begin{array}{l}1976 \\
2001 \\
1984 \\
\end{array}$ & $\begin{array}{l}2006 \\
2003 \\
2003 \\
\end{array}$ & $\begin{array}{l}76 \% / 5 \\
83 \% / 6\end{array}$ & $\begin{array}{l}\text { Sì / Havran } \\
\text { No / Havran } \\
\text { Sì / Periplum }\end{array}$ \\
\hline $\begin{array}{l}\text { Gianni } \\
\text { Biondillo }\end{array}$ & I materiali del killer & 2011 & 2015 & $78 \% / 9$ & No / Panteon \\
\hline $\begin{array}{l}\text { Fausto } \\
\text { Brizzi }\end{array}$ & $\begin{array}{l}100 \text { giorni di felicità } \\
\text { Se mi vuoi bene }\end{array}$ & $\begin{array}{l}2013 \\
2015 \\
\end{array}$ & $\begin{array}{l}2014 \\
2016 \\
\end{array}$ & $\begin{array}{l}89 \% / 104 \\
82 \% / 17 \\
\end{array}$ & $\begin{array}{l}\text { No / Fortuna Libri } \\
\text { No / Fortuna Libri }\end{array}$ \\
\hline $\begin{array}{l}\text { Andrea } \\
\text { Camilleri }\end{array}$ & $\begin{array}{l}\text { La forma dell'acqua } \\
\text { Il cane di terracotta }\end{array}$ & $\begin{array}{l}1994 \\
1996 \\
\end{array}$ & $\begin{array}{l}2002 \\
2004 \\
\end{array}$ & $\begin{array}{l}74 \% / 7 \\
80 \% / 4 \\
\end{array}$ & $\begin{array}{l}\text { No / Paseka } \\
\text { Sì / Paseka }\end{array}$ \\
\hline $\begin{array}{l}\text { Massimo } \\
\text { Carlotto }\end{array}$ & $\begin{array}{l}\text { Arrivederci amore, } \\
\text { ciao }\end{array}$ & 2005 & 2007 & $40 \% / 6$ & No / Dokořán \\
\hline
\end{tabular}

25. Nella tabella non appaiono libri di autori che non appartengono al periodo di cui ci occupiamo ma che sono stati sostenuti dal ministero italiano (p. es.: di Svevo, Montale, Ungaretti...).

26. Le case editrici che hanno ricevuto più sovvenzioni sono Argo, Paseka e Havran. Gli autori "più sostenuti” risultano Claudio Magris e Antonio Tabucchi (4x), seguiti da Eco e Vassalli (3x). 


\begin{tabular}{|c|c|c|c|c|c|}
\hline $\begin{array}{l}\text { Gianrico } \\
\text { Carofiglio }\end{array}$ & $\begin{array}{l}\text { Testimone } \\
\text { inconsapevole } \\
\text { Le perfezioni } \\
\text { provvisorie }\end{array}$ & $\begin{array}{l}2002 \\
2010\end{array}$ & $\begin{array}{l}2012 \\
2012\end{array}$ & $\begin{array}{l}75 \% / 30 \\
78 \% / 13\end{array}$ & $\begin{array}{l}\text { No / Host } \\
\text { No / Host }\end{array}$ \\
\hline $\begin{array}{l}\text { Ascanio } \\
\text { Celestini }\end{array}$ & La pecora nera & 2006 & 2012 & $87 \% / 3$ & No / dybbuk \\
\hline $\begin{array}{l}\text { Giuseppe } \\
\text { Culicchia }\end{array}$ & $\begin{array}{l}\text { Tutti giù per terra } \\
\text { Blablabla }\end{array}$ & \begin{tabular}{|l|}
1994 \\
1997 \\
\end{tabular} & $\begin{array}{l}2007 \\
2006 \\
\end{array}$ & $\begin{array}{l}73 \% / 11 \\
76 \% / 10 \\
\end{array}$ & $\begin{array}{l}\text { No / fra } \\
\text { No / dybbuk }\end{array}$ \\
\hline $\begin{array}{l}\text { Alessandro } \\
\text { D'Avenia }\end{array}$ & $\begin{array}{l}\text { Bianca come il latte, } \\
\text { rossa come il sangue }\end{array}$ & 2010 & 2014 & $76 \% / 116$ & No / Jota \\
\hline $\begin{array}{l}\text { Erri De } \\
\text { Luca } \\
\end{array}$ & Montedidio & 2001 & 2015 & $82 \% / 9$ & $\begin{array}{l}\text { Sì / Pistorius \& } \\
\text { Olšanská }\end{array}$ \\
\hline $\begin{array}{l}\text { Luca Di } \\
\text { Fulvio }\end{array}$ & $\begin{array}{l}\text { La ragazza che } \\
\text { toccava il cielo } \\
\text { La gang dei sogni }\end{array}$ & $\begin{array}{l}2013 \\
200\end{array}$ & $\begin{array}{l}2014 \\
2015\end{array}$ & $\begin{array}{l}79 \% / 239 \\
89 \% / 258\end{array}$ & $\begin{array}{l}\text { No / Knižní klub } \\
\text { No / Knižní klub }\end{array}$ \\
\hline $\begin{array}{l}\text { Umberto } \\
\text { Eco }\end{array}$ & $\begin{array}{l}\text { Baudolino } \\
\text { La misteriosa } \\
\text { fiamma della regina } \\
\text { Loana } \\
\text { Il cimitero di Praga } \\
\text { Numero zero }\end{array}$ & $\begin{array}{l}2000 \\
2004 \\
2010 \\
2014\end{array}$ & $\begin{array}{l}2001 \\
2005 \\
2011 \\
2015\end{array}$ & $\begin{array}{l}82 \% / 155 \\
77 \% / 104 \\
78 \% / 359 \\
64 \% / 141\end{array}$ & $\begin{array}{l}\text { No / Argo } 27 \\
\text { No / Argo } \\
\text { No / Argo } \\
\text { No / Argo }\end{array}$ \\
\hline $\begin{array}{l}\text { Valerio } \\
\text { Evangelisti }\end{array}$ & $\begin{array}{l}\text { Nostradamus I } \\
\text { Nostradamus II } \\
\text { Nostradamus II } \\
\text { Mistero dell'inquisi- } \\
\text { tore Eymerich }\end{array}$ & $\begin{array}{l}1999 \\
1999 \\
1999 \\
1996\end{array}$ & $\begin{array}{l}2001 \\
2002 \\
2003 \\
2005\end{array}$ & $\begin{array}{l}76 \% / 9 \\
75 \% / 8 \\
73 \% / 8 \\
65 \% / 4\end{array}$ & $\begin{array}{l}\text { No / Eminent } \\
\text { No / Eminent } \\
\text { No / Eminent } \\
\text { No / Mladá fronta }\end{array}$ \\
\hline $\begin{array}{l}\text { Oriana } \\
\text { Fallaci }\end{array}$ & $\begin{array}{l}\text { La rabbia e } \\
\text { orgoglio }{ }^{28} \\
\text { La forza della } \\
\text { ragione }\end{array}$ & $\begin{array}{l}2001 \\
2004\end{array}$ & $\begin{array}{l}2011 \\
2014\end{array}$ & $\begin{array}{l}88 \% / 8 \\
100 \% / 2\end{array}$ & $\begin{array}{l}\text { No / NLN } \\
\text { No / NLN }\end{array}$ \\
\hline $\begin{array}{l}\text { Giorgio } \\
\text { Faletti }\end{array}$ & Io uccido & 2002 & 2005 & $81 \% / 20$ & $\begin{array}{l}\text { No / Rybka } \\
\text { Publishers }\end{array}$ \\
\hline $\begin{array}{l}\text { Elena } \\
\text { Ferrante }\end{array}$ & $\begin{array}{l}\text { I giorni } \\
\text { dell'abbandono }\end{array}$ & 2002 & 2011 & $83 \% / 8$ & No / Kniha Zlín \\
\hline Dario Fo & Il paese dei mezaràt & 2002 & 2009 & $70 \% / 4$ & No / fra \\
\hline
\end{tabular}

27. Sono stati invece sovvenzionati i suoi libri di saggistica e critica (Ricerca della lingua perfetta nella cultura europea, La bustina di Minerva, Lector in fabula).

28. Tradotto dall'inglese. 


\begin{tabular}{|c|c|c|c|c|c|}
\hline Fabio Geda & $\begin{array}{l}\text { Nel mare ci sono } i \\
\text { coccodrilli }\end{array}$ & 2010 & 2011 & $81 \% / 26$ & No / Ikar \\
\hline $\begin{array}{l}\text { Paolo } \\
\text { Giordano }\end{array}$ & $\begin{array}{l}\text { La solitudine dei } \\
\text { numeri primi } \\
\text { Il corpo umano } \\
\text { Il nero e l'argento }\end{array}$ & $\begin{array}{l}2008 \\
2012 \\
2014\end{array}$ & $\begin{array}{l}2009 \\
2013 \\
2015\end{array}$ & $\begin{array}{l}88 \% / 531 \\
88 \% / 64 \\
80 \% / 70\end{array}$ & $\begin{array}{l}\text { No / Odeon } \\
\text { No / Odeon } \\
\text { No / Odeon }\end{array}$ \\
\hline Fleur Jaeggy & Proleterka & 2003 & 2005 & $40 \% / 2$ & No / Host \\
\hline Nicolai Lilin & $\begin{array}{l}\text { L'educazione } \\
\text { siberiana } \\
\text { La caduta libera } \\
\text { Il respiro del buio }\end{array}$ & \begin{tabular}{|l|}
2009 \\
2010 \\
2011
\end{tabular} & $\begin{array}{l}2011 \\
2013 \\
2014\end{array}$ & $\begin{array}{l}90 \% / 94 \\
89 \% / 47 \\
90 \% / 16\end{array}$ & $\begin{array}{l}\text { No / Paseka } \\
\text { No / Paseka } \\
\text { No / Paseka }\end{array}$ \\
\hline $\begin{array}{l}\text { Luciana } \\
\text { Littizzetto }\end{array}$ & $\begin{array}{l}\text { La principessa sul } \\
\text { pisello }\end{array}$ & 2002 & 2005 & ------------- & No / Pasienka \\
\hline $\begin{array}{l}\text { Claudio } \\
\text { Magris }\end{array}$ & $\begin{array}{l}\text { Microcosmi } \\
\text { Alla cieca }\end{array}$ & $\begin{array}{l}1997 \\
2005\end{array}$ & $\begin{array}{l}2000 \\
2011\end{array}$ & $\begin{array}{l}80 \% / 3 \\
84 \% / 5\end{array}$ & $\begin{array}{l}\text { Sì / Mladá fronta } \\
\text { Sì / Mladá fronta }\end{array}$ \\
\hline $\begin{array}{l}\text { Paolo } \\
\text { Maurensig }\end{array}$ & $\begin{array}{l}\text { La variante di } \\
\text { Lüneburg }\end{array}$ & 1993 & 2015 & $72 \% / 5$ & No / Sefer \\
\hline $\begin{array}{l}\text { Margaret } \\
\text { Mazzantini }\end{array}$ & Non ti muovere & 2001 & 2004 & $70 \% / 2$ & No / Ikar \\
\hline $\begin{array}{l}\text { Monaldi \& } \\
\text { Sorti }\end{array}$ & $\begin{array}{l}\text { Imprimatur } \\
\text { Secretum } \\
\text { Veritas } \\
\text { I dubbi di Salai }\end{array}$ & $\begin{array}{l}2002 \\
2015 \\
2015 \\
----\end{array}$ & $\begin{array}{l}2004 \\
2005 \\
2008 \\
2009\end{array}$ & $\begin{array}{l}81 \% / 18 \\
82 \% / 11 \\
87 \% / 9 \\
70 \% / 8 \\
\end{array}$ & $\begin{array}{l}\text { No / Albatros } \\
\text { No / Albatros } \\
\text { No / Albatros } \\
\text { No / Knižní klub }\end{array}$ \\
\hline $\begin{array}{l}\text { Michela } \\
\text { Murgia }\end{array}$ & Accabadora & 2009 & 2014 & $86 \% / 25$ & No / Paseka \\
\hline $\begin{array}{l}\text { Valeria } \\
\text { Parrella } \\
\end{array}$ & Per grazia ricevuta & 2005 & 2012 & $52 \% / 5$ & No / Paseka \\
\hline $\begin{array}{l}\text { Giorgio } \\
\text { Pressburger }\end{array}$ & $\begin{array}{l}\text { Storie dell'ottavo } \\
\text { distretto }\end{array}$ & 1986 & 2006 & |----------- & Sì / Sefer \\
\hline $\begin{array}{l}\text { Alessandro } \\
\text { Piperno }\end{array}$ & $\begin{array}{l}\text { Con le peggiori } \\
\text { intenzioni }\end{array}$ & 2005 & 2015 & |------------ & No / Paseka \\
\hline $\begin{array}{l}\text { Roberto } \\
\text { Saviano }\end{array}$ & $\begin{array}{l}\text { Gomorra } \\
\text { Il contrario della } \\
\text { morte } \\
\text { La bellezza e } \\
\text { l'inferno } \\
\text { Vieni via con me } \\
\text { Zero zero zero }\end{array}$ & $\begin{array}{l}2006 \\
2007 \\
2009 \\
2011 \\
2013\end{array}$ & $\begin{array}{l}2008 \\
2010 \\
2010 \\
2012 \\
2013\end{array}$ & $\begin{array}{l}78 \% / 78 \\
80 \% / 12 \\
68 \% / 5 \\
70 \% / 2 \\
80 \% / 11\end{array}$ & $\begin{array}{l}\text { No / Paseka } \\
\text { No / Paseka } \\
\text { No / Paseka } \\
\text { No / Paseka } \\
\text { No / Paseka }\end{array}$ \\
\hline Salvo Sottile & $\begin{array}{l}\text { Più scuro di } \\
\text { mezzanotte }\end{array}$ & 2009 & 2010 & $69 \% / 7$ & No / Paseka \\
\hline
\end{tabular}




\begin{tabular}{|c|c|c|c|c|c|}
\hline $\begin{array}{l}\text { Antonio } \\
\text { Tabucchi }\end{array}$ & $\begin{array}{l}\text { Il gioco del rovescio } \\
\text { Notturno indiano } \\
\text { Sostiene Pereira } \\
\text { La testa perduta di } \\
\text { Damasceno } \\
\text { Monteiro }\end{array}$ & $\begin{array}{l}1981 \\
1984 \\
1994 \\
1997\end{array}$ & $\begin{array}{l}2006 \\
2002 \\
2005 \\
2004\end{array}$ & $\begin{array}{l}87 \% \text { / } 3 \\
76 \% \text { / } 9 \\
82 \% / 21 \\
86 \% / 7\end{array}$ & $\begin{array}{l}\text { Sì / Argo } \\
\text { Sì / Argo } \\
\text { Sì / } \\
\text { Barrister\&Principal } \\
\text { Sì / Havran }\end{array}$ \\
\hline $\begin{array}{l}\text { Tiziano } \\
\text { Terzani }\end{array}$ & $\begin{array}{l}\text { Un altro giro di } \\
\text { giostra }\end{array}$ & 2004 & 2015 & $80 \% / 1$ & No / Malvern \\
\hline $\begin{array}{l}\text { Sebastiano } \\
\text { Vassalli }\end{array}$ & $\begin{array}{l}\text { La notte della } \\
\text { cometa } \\
\text { Il cigno } \\
\text { Archeologia del } \\
\text { presente } \\
\text { Un infinito numero }\end{array}$ & $\begin{array}{l}1984 \\
1993 \\
2001 \\
1999\end{array}$ & $\begin{array}{l}2014 \\
2000 \\
2006 \\
2003\end{array}$ & $\begin{array}{l}80 \% / 1 \\
78 \% \text { / } 10 \\
80 \% / 3 \\
93 \% / 6\end{array}$ & $\begin{array}{l}\text { Sì / Pistorius \& } \\
\text { Olšanská No / } \\
\text { Odeon } \\
\text { Sì / Paseka } \\
\text { Sì / Paseka }\end{array}$ \\
\hline $\begin{array}{l}\text { Giorgio } \\
\text { Vasta }\end{array}$ & Il tempo materiale & 2008 & 2011 & $66 \% / 17$ & No / Odeon \\
\hline $\begin{array}{l}\text { Mariolina } \\
\text { Venezia }\end{array}$ & $\begin{array}{l}\text { Mille anni che sto } \\
\text { qui }\end{array}$ & 2007 & 2009 & $68 \% / 8$ & No / Paseka \\
\hline $\begin{array}{l}\text { Serena } \\
\text { Vitale }\end{array}$ & Il bottone di Puskin & 1995 & 2008 & ------------- & $\begin{array}{l}\text { Sì / Pistorius \& } \\
\text { Olšanská }\end{array}$ \\
\hline Fabio Volo & $\begin{array}{l}\grave{E} \text { una vita che ti } \\
\text { aspetto }\end{array}$ & 2003 & 2010 & $71 \% / 9$ & No / Emitos \\
\hline
\end{tabular}




\title{
Allegato 2
}

\author{
Questionario Distribuito Fra Gli Studenti D’Italiano E Gli Italianisti \\ Donna / Uomo \\ Età \\ Famiglia ceca - Famiglia italo-ceca - Altro \\ Città degli studi o dell'impiego \\ (sottolinei una delle opzioni) \\ Indicativamente quanti libri legge al mese?
}

1) Quali libri della narrativa italiana degli anni Duemila ha letto? Indichi l'autore e il titolo (max. dieci)

2) Li ha letti...

in traduzione ceca

alcuni in ceco, alcuni in italiano

in lingua italiana

3) Secondo quali criteri ha scelto le Sue letture

Fanno parte del programma scolastico o universitario

Sono state consigliate dall'insegnante di lingua italiana

Suggerite da un amico e/o coetaneo

Suggerite dai genitori

Suggerite dal bibliotecario
Pubblicizzate in TV o alla radio Pubblicizzate sui social network Ha letto una recensione sulla stampa ceca Ha letto una recensione sulla stampa italiana L'ha visto in libreria Altro (precisare)

4) Quanto influisce la quarta di copertina sulla scelta del libro?

5) Conosce alcuni premi letterari italiani? Se sì, quali?

6) Ha letto dei libri perché hanno ricevuto un premio letterario? Se sì, quali?

7) Ha partecipato negli ultimi anni a degli incontri letterari con scrittori italiani? Se sì, quali?

8) Quale scrittore o scrittrice (vivente) Le piacerebbe incontrare?

9) Pensa che la letteratura italiana contemporanea abbia una specificità rispetto ad altre letterature cosiddette nazionali? Se sì, quali?

\section{DOMANDE AGLI SCRITTORI CECHI ${ }^{29}$}

1) Quali scrittori stranieri contemporanei ha letto e considera importanti per il suo lavoro?

2) Quando si dice "letteratura italiana contemporanea", le viene in mente un nome concreto? Ha letto un libro di un autore italiano pubblicato dopo il 2000? Si ricorda come l'ha scoperto

Ad 1: Non ci sono nomi citati da più di due scrittori

Ad 2: Umberto Eco, Primo Levi, Pier Paolo Pasolini, - 3x, Alberto Moravia - 2x, Antonio Tabucchi, Giorgio Vasta, Giorgio Bassani, Elsa Morante - 1x

29. Hanno risposto: Radka Denemarková, Emil Hakl, Petra Hůlová, Jiří Kratochvíl, Jan Novák, Marek Šindelka, Miloš Urban. Si sono scusati: Jiř́i Hájuícek, Tomáš Zmeškal, Jáchym Topol. Non hanno risposto: Petr Placák, Petr Šabach, Petr Borkovec, Sylva Fischerová, Tereza Boučková. 
